\title{
Alterstice
}

Revue internationale de la recherche interculturelle

International Journal of Intercultural Research

Revista International de la Investigacion Intercultural

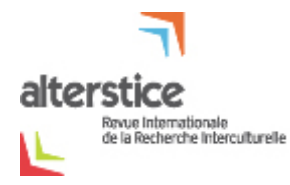

\section{Les enjeux de la survisibilisation/invisibilisation de la violence en contexte familial envers les femmes issues de l'immigration}

\section{Caroline Bouchard et Stéphanie Garneau}

Volume 3, numéro 2, 2013

Violence conjugale et diversité culturelle

URI : https://id.erudit.org/iderudit/1077523ar

DOI : https://doi.org/10.7202/1077523ar

Aller au sommaire du numéro

Éditeur(s)

Alterstice

ISSN

1923-919X (numérique)

Découvrir la revue

Citer cet article

Bouchard, C. \& Garneau, S. (2013). Les enjeux de la

survisibilisation/invisibilisation de la violence en contexte familial envers les

femmes issues de l'immigration. Alterstice, 3(2), 81-95.

https://doi.org/10.7202/1077523ar
Résumé de l'article

Dans cet article, nous proposons d'interroger la visibilité sociale - et son envers, l'invisibilité - dans la manière dont est traitée la violence en contexte familial « occidental " et « non occidental " dans les médias, en vue de réfléchir à leurs effets potentiels en termes d'intervention auprès des femmes et des filles issues de l'immigration. À partir d'une analyse critique de 95 articles du Globe and Mail, du National Post et du Toronto Star, nous montrons que les populations immigrantes font l'objet d'un excès de visibilité dans les journaux lorsqu'il est question de violence envers les femmes en contexte familial et que cet excès de visibilité a pour effet paradoxal d'invisibiliser socialement celles-là mêmes auxquelles on souhaite venir en aide. Trois conséquences potentiellement nuisibles de ce double processus de survisibilisation/invisibilisation sont discutées, avant de conclure sur des pistes de recherche et d'intervention. 
ARTICLE THÉMATIQUE

\section{Les enjeux de la survisibilisation/invisibilisation de la violence en contexte familial envers les femmes issues de l'immigration}

Caroline Bouchard $^{1}$ et Stéphanie Garneau ${ }^{1}$

\section{Résumé}

Dans cet article, nous proposons d'interroger la visibilité sociale - et son envers, l'invisibilité - dans la manière dont est traitée la violence en contexte familial " occidental » et " non occidental » dans les médias, en vue de réfléchir à leurs effets potentiels en termes d'intervention auprès des femmes et des filles issues de l'immigration. À partir d'une analyse critique de 95 articles du Globe and Mail, du National Post et du Toronto Star, nous montrons que les populations immigrantes font l'objet d'un excès de visibilité dans les journaux lorsqu'il est question de violence envers les femmes en contexte familial et que cet excès de visibilité a pour effet paradoxal d'invisibiliser socialement celles-là mêmes auxquelles on souhaite venir en aide. Trois conséquences potentiellement nuisibles de ce double processus de survisibilisation/invisibilisation sont discutées, avant de conclure sur des pistes de recherche et d'intervention.

\section{Rattachement des auteures \\ ${ }^{1}$ École de service social, Université d’Ottawa, Ottawa, Canada}

\section{Correspondance}

cbouc085@uottawa.ca

\section{Mots clés}

violence en contexte familial; analyse critique du discours; médias; invisibilité; reconnaissance

\section{Pour citer cet article :}

Bouchard, C. et Garneau, S. (2013). Les enjeux de la survisibilisation/invisibilisation de la violence en contexte familial envers les femmes issues de l'immigration. Alterstice, 3(2), xxx-xxx. 


\section{Introduction}

Selon le plus récent rapport sur la violence familiale publié par Statistique Canada (2013), plus des deux tiers (69\%) des victimes de violence familiale en 2011 étaient de sexe féminin. Pour la même année, le nombre de filles/femmes ayant subi une agression sexuelle de la part d'un membre de leur famille fut plus de cinq fois plus élevé (6 240) que le nombre de garçons/hommes (1183). Les femmes ont également subi deux fois plus d'agressions physiques en contexte familial (45 419) que les hommes (22 372). En 2011, huit fois plus de femmes ont été tuées par leur conjoint (59) que l'inverse (7).

Selon les données officielles que nous possédons, les taux de violence chez les personnes immigrantes et les minorités visibles se situeraient près de la moyenne canadienne (Statistique Canada, 2011). En 2009, le taux de violence en contexte familial chez les immigrants était en fait légèrement inférieur à la moyenne nationale, soit $4 \%$. "Des proportions semblables de membres et de non-membres de minorités visibles ont dit avoir vécu de la violence conjugale (5\% par rapport à $6 \%$ ), alors que les personnes qui ont dit être un immigrant étaient moins susceptibles de déclarer avoir été victimes de violence conjugale que ne l'étaient les non-immigrants (4\% par rapport à $7 \%$ ) " (Statistique Canada, 2011, p. 12). Toujours selon Statistique Canada (2011), environ le quart des répondants qui ont dit avoir été victimes de violence ont contacté la police. "[L]es enquêtes sur la victimisation autodéclarée démontrent que moins du quart des victimes de violence conjugale (22\% en 2009) en informent la police " (Statistique Canada, 2011, p. 8). Selon ces données, plus des trois quarts des agressions ne sont donc jamais dénoncées, tant chez les immigrants et les membres des minorités visibles que dans le reste de la population canadienne, ce qui rend difficile toute évaluation des taux réels de violence familiale. Au-delà de ces difficultés à la chiffrer, force est toutefois de constater que la violence envers les femmes en contexte familial, entendue ici comme toute forme de violence physique, sexuelle, psychologique ou économique envers les femmes et les enfants de la famille, y compris élargie (Tolan, Gorman-Smith et Henry, 2006; Dempsey, 2006), est encore aujourd'hui un problème de société important au Canada.

Dans la littérature, l'interprétation de la violence envers les femmes en contexte familial s'est longtemps faite, et se fait encore souvent, en plaçant l'oppression sexiste au cœur de l'analyse (Forman et Macleod, 1996; Hester, Kelly et Radford, 1996; Kelly et Lovett, 2004). Or, particulièrement lorsqu'il est question de migration, au moins deux autres facteurs sont importants pour comprendre la complexité de ce phénomène de violence : la déqualification professionnelle et la marginalisation économique d'une part, et le racisme et la discrimination d'autre part. En effet, plusieurs familles immigrantes se retrouvent dans des conditions socioéconomiques précaires provoquées par une déqualification professionnelle à la suite de leur arrivée au pays (Hachimi Alaoui, 2006). L'accès au marché du travail constitue souvent un obstacle de taille pour les nouveaux arrivants. Plusieurs se voient donc dans l'obligation d'occuper des postes peu qualifiés pour subvenir à leurs besoins. Cette situation constitue une source importante de stress pour les familles, ces dernières subissant une perte importante à la fois quant à leur statut social et quant à leur sécurité financière (Cardu, 2008).

En lien avec la déqualification professionnelle, la discrimination et le racisme représentent une seconde problématique de taille pour les familles immigrantes. Selon Cousineau et Bourdarbat (2009), la situation économique des immigrants s'est considérablement détériorée au cours des dernières années, en grande partie à cause du manque de sensibilisation des employeurs aux dangers de la discrimination et à un certain laxisme par rapport à l'application des lois antidiscriminatoires. En effet, l'Institut national d'études démographiques a mené une étude longitudinale qui a permis de démontrer que l'origine des immigrants avait une influence déterminante sur leur capacité à décrocher un emploi correspondant à leurs compétences. Selon cette étude, les travailleurs d'origine européenne ont peu de difficulté à trouver du travail, contrairement aux personnes originaires d'Afrique ou du Moyen-Orient (Piché, Renaud et Gingras, 2002). "L'accès au premier emploi est plus difficile pour les immigrants de l'Afrique subsaharienne, même en considérant leur capital humain et les divers cours suivis après leur arrivée. Le statut socioéconomique des emplois qu'ils détiennent après six mois est également touché par leur origine nationale, toute chose étant égale par ailleurs » (Renaud, Piché et Godin, 2003, p. 165).

Alterstice-Revue Internationale de la Recherche Interculturelle, vol. 3, $n^{\circ} 2$ 
Sans affirmer que ces facteurs puissent justifier les comportements violents, ne pas en tenir compte dans l'analyse serait négliger les sources sociales de stress, de détresse et de sentiment d'impuissance pouvant contribuer à la création d'un environnement propice à l'éclatement de la violence au sein des familles immigrantes. "Ces phénomènes peuvent placer les immigrants dans un nouveau contexte structurel violent et être vécus paradoxalement en les renforçant psychiquement ou en provoquant de la souffrance et de la fragilité. Une des manifestations en est la naissance ou l'amplification de la violence conjugale dans l'espace de la maisonnée » (Rojas Viger, 2008, p. 126).

L'approche que nous souhaitons défendre ici s'inscrit dans le sillage des théories féministes de l'intersectionnalité. Nous concevons que la violence est un phénomène complexe qui ne peut se réduire à un seul facteur d'explication dont la portée serait universellement valable. L'analyse de la violence envers les femmes en contexte familial devrait plutôt interroger simultanément les multiples formes d'oppression (sexiste, économique, raciale, etc.) au carrefour desquelles les femmes se situent et qui peuvent agir sur elles de façon particulière, selon les contextes (Kanuha, 1996; Krane, Oxman-Martinez et Ducey, 2000; Oxman-Martinez, Krane, Corbin et Loiselle-Léonard, 2002).

Dans cet article, nous proposons d'analyser la manière dont les médias traitent de la violence envers les femmes en contexte familial afin d'observer si ces diverses formes d'oppression sont analysées dans leur multiplicité et leur intersectionnalité. L'objectif derrière cette démarche est de réfléchir aux effets potentiels que peuvent avoir les modes de construction médiatique d'un problème social sur les politiques sociales et les pratiques d'intervention auprès des femmes et des filles issues de l'immigration. Pour ce faire, nous avons analysé les discours journalistiques contenus dans trois quotidiens ontariens en distinguant les propos qui concernaient la violence en contexte familial « occidental » de ceux qui concernaient la violence en contexte " non occidental ».

Si les termes "occidentaux » et " non occidentaux " ont été privilégiés en dépit de la vision dichotomique et réifiante qu'ils colportent, c'est essentiellement pour deux raisons. La première est d'ordre théorique, et renvoie au fait que nombre de chercheurs font état de la forte tendance des sociétés " occidentales " à construire leur identité collective en référence à des représentations homogènes et hégémoniques d'un Autre significatif perçu comme leur envers (Alba, 2005; Bilge, 2010a; Said, 1978). Par exemple, selon Bilge (2010b, p. 9) :

Contemporary debates over citizenship and immigrant integration are increasingly characterised by the prescriptive normativity of gender equality and sexual freedoms, articulating women's rights and gay rights to the nation to draw civilizational boundaries between Western modernity, framed as liberal and secular, and non-Western cultures, supposed to be illiberal and prone to religious fanaticism.

La seconde raison, d'ordre empirique cette fois, relève du contenu de notre matériau d'analyse. Une première lecture des articles de journaux à la source des analyses présentées ici nous a effectivement permis de confirmer cette propension à l'établissement de frontières identitaires sur la base d'une dichotomie entre un " nous" occidental et un "non-nous » qui ne l'est pas, puisqu'il s'est agi de catégories souvent mobilisées par les journalistes pour désigner la violence de l' « Autre ».

Notre appropriation de ces catégories « indigènes " ne doit toutefois pas laisser croire en leur cautionnement. Au contraire, nous verrons que le raisonnement culturaliste qui les sous-tend contient des failles ayant pour effet d'invisibiliser les autres formes d'oppression, notamment sexiste, économique et raciale, dont sont l'objet les filles et les femmes issues de l'immigration victimes de violence en contexte familial. Avant cela, nous présenterons la visibilité comme pratique sociale et exigence politique de lutte, ainsi que le rôle conféré aux médias, et donc aux discours journalistiques, dans sa production. La problématique ainsi campée, nous serons mieux à même de préciser notre stratégie de recherche ainsi que les représentations sociales de la violence en contexte familial produites et relayées dans les journaux étudiés.

\section{Discours journalistiques, représentations et visibilité sociales}

La notion de visibilité semble avoir gagné en importance lorsqu'il est question, aujourd'hui, de lutte sociale et d'action collective. Tandis que certains groupes sociaux contestent un surplus de visibilité, d'autres, par exemple les "sans-papiers", revendiquent la visibilité de leur existence et de leur cause (Siméant, 1998). Les médias 
d'information jouent un rôle majeur dans l'émergence et les modalités d'exercice des luttes pour la visibilité dans les sociétés contemporaines (Thompson, 1995; Voirol, 2005). Ils conduisent au développement de nouveaux modes d'interaction sociale qui ne nécessitent plus la coprésence dans un espace physique mais peuvent être délocalisés. Les médias, dont les journaux, constituent de fait un moyen pour les individus d'une société de se représenter des phénomènes qu'ils n'ont pas expérimentés directement. Les représentations sociales médiatisées élargissent donc le bassin de modèles de comportements auxquels un individu peut se référer et s'identifier.

Cela dit, bien que les médias multiplient les repères d'identification en donnant accès à ce qui est vécu au loin, ils contribuent tout à la fois et paradoxalement à délimiter l'horizon des possibles en imposant des "formes standardisées de représentation dans lesquelles les acteurs et les énoncés doivent s'inscrire pour apparaître » (Voirol, 2005, p. 107). En effet, les discours médiatiques sont le fruit de pratiques sociales profondément inscrites dans les contextes sociaux, politiques et institutionnels (Bassiouney, 2010; Fairclough et Wodak, 1997). Les écrits journalistiques contribuent à fabriquer des représentations et des pratiques socialement et historiquement situées, mais ils sont aussi produits par ces représentations et ces pratiques (Turner, 2011). En d'autres termes, si le journaliste est doté d'un certain pouvoir de production et de médiation de l'information, il est lui aussi façonné par des valeurs et des représentations sociales, en plus d'être impliqué dans un ensemble d'enjeux et de contraintes propres aux institutions médiatiques (Voirol, 2005). Ces jeux d'influence et de contraintes peuvent jouer un rôle de (dé)légitimation idéologique en projetant sur le public des valeurs chères à certains groupes sociaux, généralement les groupes qui se trouvent dans des positions privilégiées et qui parviennent ainsi à imposer leur point de vue (Henry et Tator, 2002). II n'est pas rare, par exemple, que les mots et les images soient choisis pour que les valeurs projetées soient dotées d'un fort potentiel de séduction, ce qui a pour effet de décrédibiliser la critique (Howitt, 1982; McGuigan, 2011). Par conséquent, les discours qui ont une portée importante sont généralement ceux qui reflètent des représentations qui jouissent déjà d'un certain succès social. Selon Pickering (2011), les médias écrits qui traitent d'enjeux contemporains reproduisent parfois des stéréotypes qui peuvent ne plus être pertinents aujourd'hui, mais qui sont encore profondément ancrés dans les représentations sociales.

Ainsi, la vision du monde d'une société peut être largement façonnée par l'angle sous lequel les médias choisissent de décrire les phénomènes qu'ils observent (Richardson, 2007). La décision des médias d'accorder peu ou pas d'attention à certains enjeux peut aussi avoir un effet très important sur les représentations sociales. On comprend dès lors que les médias d'information jouent un rôle central dans les luttes pour la visibilité. Du fait de cette participation à la construction de ce qui mérite d'être connu et de ce qui doit rester dans l'ombre, les groupements sociaux ont tout intérêt à conquérir la visibilité médiatique, non seulement pour faire entendre leurs revendications, mais aussi pour gagner la faveur de l'opinion publique et influer sur les décisions politiques.

\section{Stratégie de recherche : analyser des articles de journaux}

L'hypothèse à la source de cet article est que les populations immigrantes font l'objet d'un excès de visibilité dans les journaux lorsqu'il est question de violence envers les femmes en contexte familial et que cet excès de visibilité a pour effet paradoxal d'invisibiliser socialement certaines femmes « non occidentales ». Afin de révéler les référents symboliques produits/relayés par certains médias autour de la violence envers les femmes en contexte familial "non occidental ", nous avons retenu trois grands quotidiens publiés en Ontario : le National Post, le Globe and Mail et le Toronto Star ${ }^{1}$.

Nous avons formé deux corpus d'articles publiés entre le $1^{\text {er }}$ octobre 2003 et le $1^{\text {er }}$ octobre 2005 , le premier comprenant les articles traitant de violence envers les femmes en contexte familial « non occidental » et le second comprenant les articles traitant de la même problématique, mais en contexte familial " occidental ». Si les discours journalistiques sur la violence envers les femmes en contexte familial « occidental » ont également été analysés, c'était dans le but de mieux éclairer, en un effet de miroir, la spécificité - s'il en est une - du traitement de la violence envers les femmes lorsqu'il s'agit de familles « non occidentales ». Nous avons placé dans le corpus " non occidental » les articles dans lesquels ce vocable était utilisé ainsi que, reprenant la définition proposée par Bilge

\footnotetext{
${ }^{1}$ Pour une présentation plus détaillée de la méthodologie utilisée dans le cadre de ce projet de recherche, voir Bouchard (2011).
} 
(2010b), les articles faisant référence à un «non-nous» différent des cultures catégorisées comme « occidentales".

Pour la sélection des articles composant l'échantillon, une recherche par mots-clés fut privilégiée. Dans un premier temps, nous avons employés les mots-clés domestic violence, family violence, abusive relationship, sexual assault et abuse. Ces mots-clés ont fait ressortir peu d'articles traitant de violence en contexte familial " non occidental ", le vocabulaire utilisé par les journalistes pour traiter de cette problématique n'étant pas le même que lorsqu'il s'agit de violence au sein de familles « occidentales".

Il fut donc nécessaire d'élargir la recherche afin d'inclure les mots-clés women's rights, violence against women et de faire un tri manuel pour ne conserver que les articles abordant directement la violence en contexte familial. Afin de faciliter le tri manuel, une troisième série de recherche plus précise fut effectuée afin de cibler seulement les articles qui abordaient la question de la violence chez les familles "non occidentales ». Nous avons cherché les articles qui contenaient au moins un des mots-clés précédents ainsi qu'un des mots clés suivants: immigrant, immigration, religion, religious, culture et cultural.

Nous n'avons conservé que les articles rédigés par des journalistes, qu'ils soient de nature informative (p. ex. les faits divers) ou à saveur éditorialiste. Tous les écrits de lecteurs ont donc été écartés. De plus, les articles qui traitaient uniquement du financement de refuges d'urgence pour femmes victimes de violence conjugale ont été éliminés. Nous n'avons conservé les articles qui ont mentionné ce nouveau financement que lorsque leur auteur resituait la question dans le contexte du phénomène de la violence familiale en général.

En somme, 95 articles écrits par 73 journalistes différents ont été retenus, soit 30 articles du Globe and Mail, 26 articles du National Post et 39 articles du Toronto Star. Toutes publications confondues, 47 articles traitaient de violence envers les femmes en contexte familial " occidental » et 48 articles en contexte familial " non occidental ». La proportion des articles écrits par des hommes (47\%) était légèrement inférieure à la proportion des articles écrits par des femmes (53\%).

Le corpus d'articles sur les « non-Occidentaux » comprend à la fois les articles qui traitent de personnes vivant dans les pays " occidentaux » (31 articles) et les articles sur la violence envers les femmes en contexte familial dans les pays " non occidentaux " (17 articles). Si notre attention a également porté sur les articles de journaux portant sur la violence perpétrée envers les femmes dans les pays " non occidentaux ", c'est parce que les médias, nous l'avons vu, contribuent à extirper des événements lointains de leur contexte pour les transposer localement, dans la sphère publique. Ainsi, ce qui est véhiculé sur des événements se produisant à l'étranger vient se greffer à ce qui se passe ici, pour accroître ce qui est présent sur la scène de la visibilité et ce qui est relégué en coulisse.

Pour l'analyse des données, une analyse critique du discours a été privilégiée. " CDA [critical discourse analysis] can be defined as being fundamentally interested in analyzing opaque as well as transparent structural relationships of dominance, discrimination, power and control as manifested in language " (Wodak et Meyer, 2009, p. 10). Cette stratégie d'analyse a donc moins visé la structure linguistique des formules que la façon dont les enjeux à l'étude ont été conceptualisés dans le discours. Il s'est agi d'exposer les paradoxes du discours et de déconstruire des discours qui se présentaient souvent comme neutres alors qu'ils étaient généralement ancrés dans des enjeux de pouvoir (Blommaert, 2005; Fairclough, 2003; Toolan, 1997; Van Dijk, 2001; Van Leeuwen, 2008).

Nous avons effectué une première lecture des articles, ce qui a permis d'identifier de manière inductive les principaux thèmes abordés par les journalistes, soit les causes de la violence envers les femmes en contexte familial et les représentations sociales de la femme victime de violence et de l'homme ayant des comportements violents. Une grille permettant une analyse plus systématique des articles en fonction des thèmes ayant émergé de la lecture initiale fut utilisée lors d'une deuxième lecture (Deslauriers, 1997). II a été ainsi possible de dresser un portrait des discours journalistiques sur la problématique de la violence familiale. Les éléments propres aux discours portant sur les familles " occidentales » et " non occidentales » ont été identifiés, tout comme l'ont été les éléments communs aux deux corpus. 


\section{Psychologisation et culturalisation de la violence en contexte familial : des représentations contrastées entre les familles « occidentales » et « non occidentales »}

Les caractéristiques individuelles et psychologiques des hommes constituent le principal facteur explicatif de la violence envers les femmes ayant cours dans les familles du «nous occidental ». Nous avons repéré trois grands thèmes qui englobent les diverses représentations des causes de la violence chez l'homme " occidental » dans les trois journaux à l'étude. D'une part, la violence est présentée comme une conséquence inévitable lorsqu'une femme entretient une relation avec un homme fondamentalement violent " qui ne changera jamais ». II s'agirait donc d'une manifestation de la personnalité, voire de la nature profondément violente de certains hommes. Les journalistes transmettent alors aux lecteurs la grande cruauté dont ces hommes font preuve en décrivant précisément leurs gestes: "[...] her estranged husband beat her, pushed (her) down stairs, held a knife to her throat, punched her and chocked her, and carried gasoline into the house and threatened to burn it down " (Black, Toronto Star, 27 août 2004). Les enfants sont également les victimes de la rage de ces hommes, qui les attaquent pour se venger et faire souffrir leur ex-conjointe à la suite d'une séparation : "Children appear to be a new weapon in domestic abuse, with fathers killing them to punish their wives for leaving an abusive relationship » (Priest, The Globe and Mail, 4 mai 2005).

D'autre part, les journaux à l'étude expliquent les comportements violents d'hommes « occidentaux » en faisant fréquemment référence à la détresse psychologique dont sont victimes ces hommes. La santé mentale est donc présentée comme un élément clé dans la compréhension des causes de la violence envers les femmes en contexte familial. Un nombre important d'articles mentionnent que l'homme violent était dépressif ou qu'il avait des idées suicidaires au moment de commettre les actes: "Peter Currie, who suffered from depression, took his 2-year-old daughter, Alexis, to a secluded wood and slit her throat " (Daly, Toronto Star, 12 mars 2005). On mentionne à plusieurs reprises que la détresse psychologique doit sans équivoque être prise en considération pour déterminer le potentiel de violence d'un homme. Parallèlement, on indique que ceux qui sont diagnostiqués avec un trouble de santé mentale à la suite d'une évaluation psychiatrique tendent à recevoir des peines allégées: "[T]hey can often show themselves to have been in emotional distress [and] all of those things would tend to mitigate the severity of the sentence " (Wattie et Beauchesne, National Post, 7 juillet 2004).

On considère enfin que dans un nombre important de cas de violence familiale, il s'agit de drames qu'on ne s'explique pas. Impossibles à prévoir, les meurtres ou tentatives de meurtre d'un homme sur une femme ou ses enfants provoquent une onde de choc auprès des proches qui n'arrivent pas à comprendre comment l'homme en est arrivé à poser un tel geste : "Inevitably, most who knew the family have expressed astonishment at the turn of events, even close neighbors saying they would have never dreamed such a thing possible from such an apparently normal home " (Coyle, Toronto Star, 27 août 2004). On considère souvent que la violence ne correspond pas à la vraie nature de l'homme qui a commis le crime puisqu'on fait état de la grande valeur de l'individu: "Professionally, he was an expert of climate change who spoke at panels and forums. Away from the office, he was a bodybuilder with a love of outdoor sports " (Campbell, National Post, 15 février 2005). Les gestes violents posés par l'homme, sans que l'on comprenne vraiment pourquoi, sont donc présentés comme le résultat d'une relation qui a mal tourné : "Dr. Halton and Mr. Coutts met one year before her death through a mutual friend, and it was a love-at-first-sight story that turned into abuse and ended in death » (Campbell, National Post, 15 février 2005).

En contrepartie, les articles analysés interprètent très majoritairement la violence envers les femmes en contexte familial " non occidental » à travers un prisme culturaliste, c'est-à-dire en rapportant les comportements violents, en un lien de causalité directe, à l'appartenance culturelle. Les causes individuelles de la violence, comme la détresse psychologique ou la dynamique relationnelle au sein d'un couple, n'apparaissent que dans l'un des articles. Tous les autres rapportent les comportements violents des hommes " non occidentaux " à leur culture ou à leur religion d'appartenance. De fait, les hommes « non occidentaux » violents sont dépeints comme perpétuant des traditions archaïques. Selon plusieurs journalistes, la manifestation la plus évidente de cet archaïsme serait le maintien de lois vieilles de plusieurs centaines d'années: "Florin Cioaba is a man who understands his community's fragile place in the world. He appeared on Romanian television last Tuesday to defend the wedding. "As a father, I know what is good for my kids", he declared. "There are some laws that have to be respected. We Roma have a tradition to marry our children when they are minors" " (Wiwa, The Globe and Mail, 4 octobre 2003).

Alterstice - Revue Internationale de la Recherche Interculturelle, vol. 3, $n^{\circ} 2$ 
Ce maintien des valeurs traditionnelles dans les sociétés «non occidentales » est un thème récurrent. Dans plusieurs pays étrangers, une partie de la population aurait adopté un style de vie moderne, mais refuserait de laisser de côté ses valeurs traditionnelles, que l'on considère empreintes de sexisme et de patriarcat: "Though many of us lead a comfortable life in the 21st century - driving a car, using a cellphone, watching satellite TV - the mindset of many Saudi males is that women are useful as sex objects or for obeying orders " (Vallis, National Post, 17 avril 2004). On explique aussi la violence des hommes immigrants par le fait qu'ils amènent au pays d'accueil des éléments de cette culture " non occidentale » : "Some women who flee abuse in their country of origin end up being preyed on by others in their own ethnic communities in Canada » (Keung, Toronto Star, 3 août 2004).

Plusieurs articles mentionnent la volonté de réforme de certaines sociétés "non occidentales ", mais ces changements sont présentés comme étant très difficiles à réaliser car ces sociétés sont perçues comme étant divisées entre les aspirations à la modernité et les traditions : "In Chile, where only one in three women work and divorce was only legalized last year, strong conservatism and rampant chauvinism was partly to blame keeping abusers out of the criminal justice system, women's groups say » (Ross, The Globe and Mail, 30 septembre 2005). "[T]he country struggles to reconcile ancient tribal and Islamic traditions with modern day values " (Denyer, National Post, 13 décembre 2003). S'il existe encore de telles lois, c'est donc que les pays " non occidentaux" n'ont pas encore « évolué » suffisamment pour laisser de côté certaines coutumes violentes du passé.

Quoique l'archaïsme des sociétés «non occidentales » semble être le principal prisme par lequel les journalistes interprètent la violence chez les étrangers, les journalistes de notre corpus se sont aussi intéressés à l'influence de la religion musulmane, considérée comme plus violente que les autres religions, sur les comportements violents des hommes. Plusieurs considèrent la religion musulmane comme étant plus violente à cause de sa nature profondément patriarcale : "The one common denominator is that it is strongly patriarcal » (Wente, The Globe and Mail, 29 mai 2004). Les imams qui ont immigré dans les pays "occidentaux " sont souvent accusés de puiser dans les fondements d'une religion violente pour permettre aux hommes d'abuser de leur femme. On rapporte à quelques reprises la publication d'un livre écrit par un imam qui explique aux hommes comment punir leurs femmes lorsqu'elles désobéissent: " [V]erbal warnings followed by a period of sexual inactivity could be used to discipline a disobedient wife. If that failed, he argued that, according to Islamic law, beating could be judiciously administered »(Wilkinson, National Post, 29 septembre 2005). Selon plusieurs des articles étudiés, les imams contribueraient donc à assurer l'impunité des hommes lorsqu'ils commettent des actes de violence. Selon plusieurs journalistes, puisque la religion musulmane est foncièrement violente, les femmes qui sont croyantes sont contraintes d'accepter la violence qu'elles vivent. Plusieurs se résigneraient ainsi à garder le silence: " [I]t is difficult for us to speak out because we are practicing, pro-faith Muslims " (Hurst, Toronto Star, 22 mai 2004). Le fait de vouloir vivre selon les principes de sa religion, lorsque celle-là encourage la violence, aurait pour conséquence d'augmenter la vulnérabilité des femmes : "their options were already circumscribed, not by poverty or patriarchy, but by their own desire to live within their faith » (Coyne, National Post, 17 septembre 2005).

Qu'ils traitent de la violence en contexte familial " occidental » ou en contexte familial " non occidental », rares sont les articles qui prennent en considération la situation de pauvreté dans laquelle vivent certaines familles pour mieux comprendre les raisons de la violence des hommes. Dans les articles qui traitent des familles " occidentales", le stress causé par une situation financière difficile n'est mentionné que par trois journalistes comme étant un facteur pouvant mener à la violence conjugale. Graeme Smith, du Globe and Mail, est l'un d'entre eux. Il présente les difficultés économiques récurrentes que vivent les agriculteurs au Canada et les conséquences sur la vie familiale: "Wives take second jobs and tend market gardens to help their families survive, making their husbands feel inadequate. Wives leave their husbands for easier lives. Husbands unleash their frustration on their families, leading to abuse and divorce" (8 septembre 2004). La grande majorité des articles qui aborde la problématique de la pauvreté s'intéresse plutôt aux difficultés économiques que vivent les femmes une fois qu'elles ont quitté un conjoint violent et le manque de ressources auquel elles doivent faire face : "Many women who leave violent partners have no financial independance and are not able to pay for transportation to get to the services they need " (Huffman, Toronto Star, $1^{\text {er }}$ juin 2005). En ce qui concerne le corpus des articles portant sur les familles "non occidentales", les allusions à une situation socioéconomique difficile sont encore plus rares, puisqu'un seul des articles fait un lien entre la pauvreté et la violence conjugale : "Ekuwa Smith, author of the justice department-funded study, said economic adversity doesn't help » (Keung, Toronto Star, 8 mars 2004). Les 
journalistes suggèrent plutôt que des hommes "non occidentaux » posent des gestes violents qui prennent racine dans leur culture ou, lorsqu'il s'agit d'hommes de confession musulmane, leur religion.

La violence n'est certes pas l'apanage des familles défavorisées. Mais en omettant de prendre également en compte les facteurs sociaux associés à la violence conjugale, les discours journalistiques que nous avons analysés contribuent à réduire le phénomène ou bien à un désordre psychologique (familles " occidentales ") ou bien à une culture et à une religion inadaptées aux temps modernes (familles "non occidentales »). Pourquoi les hommes violents "non occidentaux" ne seraient-ils pas affectés également, comme cela est avancé pour les hommes "occidentaux", d’un trouble de santé mentale? Les regards croisés entre familles " occidentales" et "non occidentales " sur la manière dont cette forme de violence est médiatisée dans les trois quotidiens ontariens illustrent le traitement différencié (en l'occurence culturaliste) de la violence dans les familles «non occidentales ${ }^{2}$. Nous aborderons maintenant les failles du raisonnement qui sous-tend cette lecture particulière.

\section{Les failles d'une lecture culturaliste de la violence en contexte familial « non occidental »}

Cette analyse critique des discours journalistiques dans trois quotidiens anglophones ontariens à propos de la violence perpétrée envers les femmes en contexte familial «non occidental » ne doit pas laisser penser que nous nions l'existence d'un tel phénomène. Certaines sources rapportent en effet que plus du tiers des 4000 causes entendues devant la cour municipale du Québec sont le fait de personnes issues des communautés ethniques, la plupart du temps d'immigration récente (Zorbas, 2002). D'aucuns affirment que ces chiffres pourraient être encore plus élevés en raison de la peur et de la méconnaissance des services d'aide existants, lesquelles empêcheraient certaines femmes immigrantes violentées d'y recourir (Agnew, 1998). Cela étant dit, rappelons tout de même que l'Enquête sociale générale de Statistique Canada (2011) sur la violence en milieu familial relate que si $35 \%$ des homicides au Canada sont commis en contexte familial et que les femmes sont trois fois plus "à risque » d'en être victimes, ces cas de violence ne sont pas proportionnellement plus fréquents dans les familles des groupes minoritaires. Les données statistiques ont une certaine "labilité » et nécessitent, pour plus de rigueur, que nous nous assurions de leur comparabilité. Quels sont les chiffres récoltés par les services de police? Ceux des tribunaux ? Pour quels actes de violence ? Et pour quelle ville, région ou province?

Le propre de notre propos ne tient de toute façon pas dans ce calcul empirique autour de l'ampleur avérée ou non du phénomène de la violence dans les familles "non occidentales ". Nous considérons que plusieurs formes de violence existent bel et bien dans certaines de ces familles et nous laissons à d'autres le soin d'en dresser le portrait. Si nous appréhendons de manière critique le discours journalistique dont nous avons fait ici l'analyse, c'est parce qu'il comporte trois défaillances principales sur lesquelles nous aimerions attirer l'attention car elles peuvent avoir, à notre avis, des effets pernicieux sur les filles et les femmes victimes de violence.

La première faille concerne les assises théoriques du concept même de culture ainsi mise de l'avant, lesquelles sont erronées, comme le montrent amplement depuis nombre d'années les études anthropologiques et sociologiques (Poutignat et Streiff-Fenart, 1995). Si la culture a bien des effets structurants sur les manières de penser et d'agir des individus - d'ailleurs autant chez les «Canadiens » que chez les membres des autres sociétés -, elle n'est jamais complètement homogène ni immuable dans le temps et dans l'espace (Barth, 1995; Bayart, 1996). Lorsqu'on se laisse aller à une pensée culturaliste, en postulant par exemple qu'une culture ou une religion est fondamentalement violente, il est difficile, si on pousse au bout le raisonnement, de ne pas affirmer dans la foulée que tous les hommes appartenant à cette culture ont une tendance, depuis toujours et quelle que soit la société dans laquelle ils vivent, à être violents, et que toutes les femmes ont une propension à en être victimes ${ }^{3}$. Ce serait également prétendre que lorsque les hommes ne se rendent pas jusqu'aux actes, ce serait au prix d'une énorme lutte contre nature. En culturalisant la violence, c'est tout le groupe qui en est porteur qui est visé et minoré. Et, en homogénéisant culturellement, on naturalise la différence culturelle (Fassin et Fassin, 2006). Or le « contenu »

\footnotetext{
${ }^{2}$ Le traitement journalistique du phénomène de la violence en contexte familial « occidental » n'est pas non plus dénué d'effets $d^{\prime}$ (in)visibilité sociale. Pour une analyse de l'invisibilisation du patriarcat dans les sociétés " occidentales » et de ses atteintes potentielles aux revendications féministes, voir Bouchard (2011).

${ }^{3}$ De plus, cela laisse à penser que seuls les autres sont porteurs d'une culture particulière, comme si le " nous » était dépourvu de toute appartenance culturelle.
}

Alterstice - Revue Internationale de la Recherche Interculturelle, vol. 3, $n^{\circ} 2$ 
d'une culture prend toujours forme dans un contexte interactionnel, politique, historique, économique et social déterminé (Bayart, 1996).

Cette réduction « naturalisante » a pour cause et effet malencontreux l'évacuation des processus sociaux à l'origine de plusieurs situations de violence en contexte familial (comme la pauvreté, la discrimination, la stigmatisation et le racisme), ce qui constitue la deuxième faiblesse d'une explication culturaliste de la violence. Une telle appréhension du phénomène de la violence faite aux femmes en contexte familial a des effets politiques condamnables en détournant l'attention des facteurs structurels sur lesquels il conviendrait d'agir socialement pour améliorer les conditions de vie socioéconomiques de pans entiers de familles ainsi que pour sensibiliser aux différences culturelles. Le processus de naturalisation repéré dans les discours journalistiques n'épargne d’ailleurs pas la violence en situation familiale " occidentale ", puisque nous avons vu que la violence y faisait le plus souvent l'objet d'une explication individuelle et biopsychologique. Le détournement de l'action publique des facteurs sociaux vecteurs de violence affecte donc aussi l'intervention auprès de femmes qui ne sont pas en situation migratoire.

La troisième faille du raisonnement culturaliste produit et relayé dans les journaux étudiés concerne les effets nuisibles concrets qu'il peut avoir sur les populations mêmes auxquelles on prétend vouloir venir en aide : les filles et les femmes issues des minorités. II produit un double processus de survisibilisation/invisibilisation sur lequel nous aimerions maintenant nous attarder.

\section{Survisibilisation et invisibilisation : les conséquences nuisibles des représentations homogénéisantes de la violence en contexte familial « non occidental » sur les femmes et filles issues de l'immigration}

Lorsqu'il est question de violence en contexte familial, nous l'avons vu, les groupes minoritaires «non occidentaux " font l'objet d'un traitement spécifique en ce que les actions de brutalité sont le plus souvent expliquées par la culture et la religion alors que ce n'est pas le cas pour les familles dites "occidentales ». Cette attention publique particulière produit une survisibilité des populations issues de l'immigration, puisque ces dernières deviennent partie prenante d'un univers culturel réifié, défini à gros traits comme étant celui du patriarcat, de la barbarie et de l'archaïsme. Cet excès de visibilité sociale s'apparente à un « déni de reconnaissance ॥ au sens de Renault (2007, p. 202), puisque l'Autre est ainsi renvoyé à ce qu'il a de plus étranger à soi et, surtout, puisque cet Autre se résume à cette représentation globale et forcément stéréotypée. Pis encore : en rapportant les comportements de certains hommes "non occidentaux " violents à leur culture ou religion d'appartenance, en faisant donc de cette culture ou de cette religion une entité unique et homogène, ce sont ses autres membres, non violents, qui sont alors dénués d'existence. La question de la visibilité ne doit pas être confondue ici avec celle de la reconnaissance. Tandis que la visibilité sociale correspond aux « modes d'apparition mutuels par lesquels les acteurs sociaux viennent à exister les uns pour les autres » (Voirol, 2005, p. 112), la reconnaissance appelle une relation intersubjective qui, par la validation d'une reconnaissance réciproque entre deux individus ou deux groupes d'individus, leur permettent de se constituer en sujet et de développer un rapport positif à soi (Honneth, 1992; Renault, 2004). Nous comprenons dès lors qu'un individu ou un groupe d'individus invisible n'intervient donc aucunement dans cette interaction, et qu'il est donc d'emblée rejeté de toute possibilité de reconnaissance. En d'autres mots, sa reconnaissance ne lui est même pas déniée : il est tout simplement dénué d'existence.

Trois conséquences nuisibles pour les filles et femmes issues de l'immigration et violentées en contexte familial peuvent potentiellement découler de la survisibilisation dont sont l'objet, dans les discours analysés, la culture et la religion des familles « non occidentales » qui connaissent de la violence masculine. L'excès de visibilité des identités culturelles minoritaires, lorsqu'il est question de violence faite aux femmes en contexte familial, peut avoir pour première conséquence inattendue d'encourager le recours à un " prisme culturaliste » comme agent disculpant les hommes, avec pour effet subséquent de mépriser socialement les femmes victimes de violence. En effet, la tendance à la culturalisation des rapports sociaux et la prégnance de représentations stéréotypées de la violence faite par des hommes issus des minorités racisées sur « leurs » femmes, dont les journalistes se sont fait le relais dans notre corpus d'articles, peut paradoxalement masquer un autre axe de minoration : celui basé sur le genre. Dans un article portant sur les représentations de la violence contre les femmes des minorités dans le système de 
justice canadien, Bilge (2006a) rapporte des cas de jugement où un homme d'une minorité culturelle a vu sa peine réduite pour un crime commis sur une femme de la même appartenance culturelle sous le prétexte de mœurs et coutumes présumées de cette culture en matière de relations hommes-femmes et de sexualité. Si les affaires de "meurtres d'honneur » ont rarement bénéficié d'un traitement d'exception basé sur l'« information culturelle » dans les tribunaux canadiens, dans d'autres actes de violence, notamment des cas de viol, la culture a pu servir de circonstances atténuantes au nom du respect de la diversité culturelle, déniant dans la foulée toute reconnaissance à l'identité de genre (et au statut de victime de sévices sexuels) aux femmes de ces mêmes groupes culturels. La femme victime de violence d'un ou de plusieurs hommes disparaît derrière son identité culturelle.

Cette survisibilisation des groupes minoritaires "non occidentaux » peut produire non seulement un déni de reconnaissance de la femme " non occidentale », mais elle a directement des effets d'invisibilisation des femmes et des filles issues de l'immigration (ainsi d'ailleurs que d'autres personnes vulnérables en leur sein, comme les personnes non hétérosexuelles, les enfants de sexe masculin, etc.). Ce sont les femmes "non occidentales " qui souhaiteraient s'élever contre la violence dont elles peuvent être victimes sans avoir à renoncer à leur identité culturelle ou religieuse qui sont ainsi invisibilisées. La deuxième conséquence plausiblement nocive de ce double processus de survisibilisation/invisibilisation est donc de priver de voix, $d$ 'isoler et d'enfermer toujours plus avant des femmes et filles issues de l'immigration victimes de violence dans une situation familiale où elles sont en danger.

La question de la violence faite aux femmes en contexte d'immigration ne manque pas de rappeler l'opposition entre le féminisme et le multiculturalisme, qui a lieu autant dans les débats publics que dans les discussions $d^{\prime}$ intellectuels ${ }^{4}$. Les termes de cette opposition sont d'ailleurs entièrement contenus dans le point que nous venons à l'instant de développer en recourant à ces cas-limites de la jurisprudence, puisque le droit de pratiquer sa culture et sa religion, que veulent notamment protéger les politiques sur le multiculturalisme, peut bel et bien entrer en conflit avec les droits et les revendications des femmes dans leur lutte pour l'égalité des sexes. Cette manière antinomique de poser le problème risque toutefois de fausser les termes de la réflexion car, en forçant à faire un choix - en cas de conflit, préséance à l'égalité des sexes ou préséance à l'égalité des cultures ? -, l'identité culturelle et l'identité de genre apparaissent comme des entités mutuellement exclusives. De ce fait, lorsqu'une culture ou une religion est représentée dans les médias comme foncièrement violente, souvent par le biais de ses représentants masculins, cela place les femmes qui ont cette culture ou cette religion en partage et qui sont victimes de violence de la part de "leurs » hommes devant l'obligation de faire un choix déchirant : ou bien elles renoncent à leur identité culturelle et religieuse "à la source » de leur malheur pour embrasser la perspective d'un féminisme "classique " (libéral et universaliste, pour ne pas dire blanc et occidental) et défendre leur identité de femme meurtrie, ou bien elles ne veulent pas le faire (souvent parce qu'elles sont également sensibles au racisme que vivent "leurs" hommes ou parce qu'elles se disent et se sentent appartenir à la culture ou à la religion décriée) et elles sont alors condamnées à subir en silence leur malheur (Bilge, 2006b). Il y a certes des femmes qui prennent la première décision, comme certaines qui, originairement de confession musulmane, prennent la parole dans l'espace public pour condamner le caractère " archaïque » de cette religion. Mais pour les autres, celles qui souhaiteraient combattre la violence dont elles sont victimes en raison de leur identité de genre tout en n'ayant pas à renier, trahir ou pourfendre leur identité culturelle ou religieuse, leur voix n'est pas entendue. L'évocation même de leur existence paraît une aberration. Sans voix, elles sont confinées au silence et au repli sur soi et sont ainsi maintenues dans le danger. En effet, comment, dans de telles circonstances, leur serait-il possible de trouver, voire même de chercher, les services d'aide adaptés ? Sokoloff et Dupont (2005) soulignent d'ailleurs qu'en raison de la peur du traitement injuste que pourrait recevoir leur conjoint, certaines femmes choisissent de ne pas aller chercher de l'aide.

\footnotetext{
${ }^{4}$ Le débat a notoirement opposé Susan Moller Okin (1997) à Chandran Kukathas (2001), mais des critiques se sont fait entendre dès la parution du texte de Okin dans le numéro de la Boston Review où il a été publié en 1997. II a, depuis, largement dépassé la sphère universitaire.
} 
Enfin, la troisième conséquence potentielle de cette survisibilité d'une « culture » médiatisée comme violente et de l'invisibilité de ces femmes et filles issues de l'immigration qui souhaiteraient dénoncer la violence dont elles font l'objet en contexte familial sans avoir à renier une partie de soi est la production d'un rapport négatif à soi. Selon Voirol (2005, p. 117-118) :

Les acteurs invisibles sont privés d'attention, ne font pas l'objet d'une quelconque considération, pas même celle de la stigmatisation; ils se trouvent exclus, non seulement des relations de reconnaissance, mais des relations tout court. Autrement dit, il y a une forme de mépris extrême qui passe par le silence et l'invisibilité dans l'espace d'apparition publique et qui surpasse de loin les formes de mépris s'exprimant par l'insulte, le dénigrement et la dévalorisation.

Si l'invisibilité sociale peut conduire à des "luttes pour la visibilité », à sortir du silence pour dénoncer publiquement et collectivement le tort vécu, force est de concéder que la population qui nous intéresse ici se trouve dans une situation d'extrême vulnérabilité. L'alternative à une lutte pour la visibilité - difficile à engager s'il faut pour cela affronter la honte de son identité culturelle ou nier une partie de soi - peut alors être la " mortification identitaire ", laquelle se donne à voir de diverses manières : désocialisation extrême et réduction du sens de l'existence conduisant à la seule satisfaction des besoins primaires, haine de soi accompagnée de comportements autodestructeurs ou encore violence envers autrui ou son environnement (Renault, 2004). Ces femmes à l'identité personnelle dépréciée, à la fois comme femme par "leurs » hommes et comme culturellement autre par la société majoritaire, risquent de développer divers troubles les condamnant toujours plus avant à l'isolement. Si les causes de la violence des hommes en contexte familial ne sont jamais réductibles à un phénomène biopsychologique, leurs liens possibles en termes de manifestations psychologiques chez celles qui en sont victimes ne peuvent être niés (Golding, 1999; Humphreys, Regan, River et Thiara, 2005; Humphreys et Thiara, 2003).

\section{Conclusion : quelles pistes pour les journalistes, les intervenants sociaux et les chercheurs?}

En survisibilisant certaines cultures ou religions comme causes de la violence en contexte familial «non occidental ", donc en médiatisant les populations - prioritairement les hommes - appartenant à ces cultures ou religions comme fondamentalement ou potentiellement violentes, ce sont les individus non violents de ces mêmes groupes qui se trouvent à être niés dans leur existence. Parmi ces individus invisibilisés se trouvent celles-là mêmes qui ont besoin d'aide, les filles et les femmes victimes de brutalité dans leur famille. Mais il se trouve une frange de cette population qui est encore plus dénuée d'existence : les femmes et les filles victimes de violence dans leur famille qui voudraient dénoncer leur(s) bourreau(x) tout en pouvant légitimement et fièrement continuer de revendiquer leur appartenance culturelle ou religieuse.

De là à affirmer que ces processus de survisibilisation/invisibilisation se vérifient dans les pratiques d'intervention sociale, il y a un pas que nous ne franchirons pas. Si les représentations sociales relayées dans les médias circulent également dans la société, en amont et en aval de leur diffusion, on ne peut présumer de l'ampleur et de la nature de l'influence des discours journalistiques sur la population (Charaudeau, 1997; Dittmar et von Stutterheim, 1985; Howitt, 1982; McGuigan, 2011; Wilson, Gutiérrez et Chao, 1995). Trois raisons principales peuvent être convoquées pour inviter à la prudence quant au lien à établir entre les discours médiatiques et les pratiques d'intervention.

Premièrement, les individus sont dotés de capacité de jugement et d'interprétation critique de ce qu'ils lisent, voient ou entendent (Manjarrés, 2007). Affirmer que les médias exercent un pouvoir unilatéral sur le public, ce serait partir du postulat contestable que ce dernier est une coquille vide et se laisse remplir inéluctablement, incapable de réflexivité.

Deuxièmement, la population civile n'est pas une et uniforme. Si à tout hasard il était vérifié par un sondage que les représentations de la violence en contexte familial «non occidental " qui sont véhiculées dans les journaux trouvent une résonnance dans l'opinion publique, il resterait encore à vérifier s'il en va de même dans le monde spécifique de l'intervention auprès des femmes violentées. La société civile ne forme pas une masse indifférenciée, et elle doit plutôt être appréhendée comme un ensemble formé d'univers normatifs pluriels. 
Enfin, des sociologues et politologues de l'action publique remettent depuis plusieurs années en cause le postulat selon lequel les idées influenceraient les pratiques (Desage et Godard, 2005; Musselin, 2000). Même si le discours auquel se réfèrent eux-mêmes les " acteurs de terrain " sur leurs pratiques peut sembler faire écho aux catégories sociales médiatisées dans les journaux, rien ne nous empêche d'émettre l'hypothèse qu'il puisse y avoir des décalages, voire des contradictions, entre les idées et leur traduction en actions (Oxman-Martinez et Krane, 2005). Il devrait être du ressort de la recherche que d'identifier les cadres concrets, idéels tant que matériels, qui modulent les interventions telles qu'elles se donnent vraiment à voir plutôt que de supposer que ces dernières s'inspirent directement des catégories disponibles dans l'espace médiatisé.

Si ces trois éléments doivent être pris en considération et nous inciter à la prudence quant aux liens à faire entre représentations médiatisées dans les journaux et pratiques concrètes d'intervention, ils invitent néanmoins à une profonde réflexion sur les méthodes de recherche, les pratiques d'intervention et le travail journalistique. Ils appellent en effet les chercheurs à mettre en place des dispositifs de recherche ethnographique facilitant l'appréhension de l'intervention en situation, de manière à révéler les catégories normatives effectives tout autant que les configurations de relations, jamais dépourvues de pouvoir, qui concourent à produire telles ou telles pratiques d'intervention et leurs effets. Ils invitent également les concepteurs de politiques sociales et les intervenants à réfléchir, dans leurs pratiques quotidiennes, à la part d'invisibilité contenue dans les programmes d'intervention et les politiques sociales et à la possibilité qu'elle ait des conséquences sur les acteurs individuels et collectifs auxquels ils viennent en aide.

Sans postuler que la presse écrite dans son ensemble adopte une lecture culturaliste du phénomène, puisque les discours journalistiques sont également un lieu où sont discutées des visions du monde en opposition, nos réflexions invitent les journalistes qui seraient tentés par le prisme culturaliste à réinjecter dans leurs analyses la complexité des facteurs sociaux qui entrent dans la production de la violence envers les femmes en contexte familial. Même si les processus culturels structurent en partie les façons de penser (et de commettre) la violence, d'ailleurs tant dans les sociétés "non occidentales » que dans les sociétés " occidentales ", la violence ne se réduit jamais à la culture vue comme une substance. En adoptant une lecture plus structurelle de la violence envers les femmes en contexte familial " non occidental », les différents acteurs des médias préviendraient les processus, même non intentionnels, de survisibilisation et d'invisibilisation culturelles identifiés dans cet article. C'est en reconnaissant l'imbrication des diverses formes d'oppression qu'on pourra éviter de vulnérabiliser davantage les filles et les femmes issues de l'immigration qui sont violentées en contexte familial.

\section{Références bibliographiques}

Agnew, V. (1998). In search of a safe place: abused women and culturally sensitive services. Toronto : University of Toronto Press.

Alba, R. (2005). Bright vs. Blurred boundary: Second-generation assimilation and exclusion in France, Germany, and the United States. Ethnic and racial studies, 28(1), 20-49.

Barth, F. (1995 [1969]). Les groupes ethniques et leurs frontières. Dans P. Poutignat et J. Streiff-Fenart, Théories de l'ethnicité (p. 203-249). Paris : Presses universitaires de France.

Bassiouney, R. (2010). Arabic and the media. Linguistic analysis and applications. Boston : Brill.

Bayart, J.-F. (1996). L'illusion identitaire. Paris : Fayard.

Bilge, S. (2006a). Behind the "culture" lens. Judicial representations of violence against minority women. Canadian woman studies, 25(1-2), 173-180.

Bilge, S. (2006b). Le dilemme genre/culture ou comment penser la citoyenneté des femmes minoritaires au-delà de la doxa féminisme/multiculturalisme ? Communication présentée au colloque du Conseil du statut de la femme Diversité de foi / Égalité de droits, Montréal, Canada. www.csf.gouv.qc.ca/modules/fichierspublications/fichier-28-353.pdf

Alterstice-Revue Internationale de la Recherche Interculturelle, vol. 3, $n^{\circ} 2$ 
Bilge, S. (2010a). “...alors que nous, Québécois, nos femmes sont égales à nous et nous les aimons ainsi” : la patrouille des frontières au nom de l'égalité de genre dans une "nation" en quête de souveraineté. Sociologie et sociétés, 42(1), 197-226.

Bilge, S. (2010b). Beyond subordination vs. resistance: an intersectional approach to the agency of veiled muslim women. Journal of intercultural studies, 31(1), 9-28.

Blommaert, J. (2005). Discourse: key topics in sociolinguistics. Cambridge : Cambridge University Press.

Bouchard, C. (2011). Les représentations sociales de la violence au sein des familles occidentales et nonoccidentales. Analyse critique comparative du discours journalistique lors du débat autour de la mise sur pied de tribunaux d'arbitrage islamiques pour les litiges familiaux en Ontario (mémoire de maîtrise, Université d'Ottawa, Canada).

Cardu, H. (2008). Construction identitaire professionnelle et interaction en contexte de transition culturelle : l'étude d'un cas. Connexions, 89(1), 171-180.

Charaudeau, P. (1997). Le discours d'information médiatique. La construction du miroir social. Paris: Nathan.

Cousineau, J.-M. et Bourdabat, B. (2009). La situation économique des immigrants au Québec. Relations industrielles, 64(2), 230-249.

Dempsey, M. (2006). What counts as domestic violence? a conceptual analysis. William and Mary Journal of women and the law, 12(2), 300-333.

Desage, F. et Godard, J. (2005). Désenchantement idéologique et réenchantement mythique des politiques locales. Revue française de science politique, 55(4), 633-661.

Deslauriers, J.-P. (1997). L'induction analytique. Dans J. Poupart et collab. (dir.), La recherche qualitative. Enjeux épistémologiques et méthodologiques (p. 125-152). Montréal : Gaëtan Morin éditeur.

Dittmar, N. et von Stutterheim, C. (1985). On the discours of immigrant workers: interethnic communication and communication strategies. Dans T. Van Dijk (dir.), Handbook of discourse analysis (p. 125-152). Montréal : Academic Press.

Fairclough, N. (2003). Analysing discourse: textual analysis for social research. New York : Routeledge.

Fairclough, N. et Wodak, R. (1997). Critical discourse analysis: An overview. Dans T. Van Dijk (dir.), Discourse studies, a multiplinary introduction (p. 67-97). Londres : Sage.

Fassin, D. et Fassin, E. (2006). Conclusion. Éloge de la complexité. Dans D. Fassin et E. Fassin (dir.), De la question sociale à la question raciale? Représenter la société française (p. 249-259). Paris : La Découverte.

Forman, J. et Macleod, J. (1996). Working against violence against women and children. Dans H. Bradby (dir.). Defining violence: understanding the causes and effects of violence (p. 27-43). Aldershot et Brookfield : Avebury.

Golding, J. (1999). Intimate partner violence as a risk factor for mental disorders: a meta-analysis. Journal of family violence, $14(2), 99-132$.

Hachimi Alaoui, M. (2006). "Carrière brisée », « carrière de l’immigrant " : le cas des Algériens installés à Montréal. Les Cahiers du Gres, 6(1), 111-122.

Henry, F. et Tator, C. (2002). Discourse of domination: racial biais in the canadian English-language press. Toronto : University of Toronto Press.

Hester, M., Kelly, L. et Radford, J. (dir.) (1996). Women, violence and male power. Buckingham : Open University Press.

Honneth, A. (1992). La lutte pour la reconnaissance. Paris : Le Cerf.

Howitt, D. (1982). The mass media and social problems. Toronto : Pergamon Press. 
Humphreys, C., Regan, L., River, D. et Thiara, R. (2005). Domestic violence and substance use: tackling complexity. British journal of social work, 35, 1303-1320.

Humphreys, C. et Thiara, R. (2003). Domestic violence and mental health: "I call it symptoms of abuse". British journal of social work, 33(2), 209-226.

Kanuha, V. (1996). Domestic violence, racism and the battered women's movement in the United States. Dans J. Eldeson et Z. Eisikovits (dir.), Future interventions with battered women and their families (p. 34-50). Thousand Oaks, CA : Sage Publications.

Kelly, L. et Lovett, J. (2004). What a waste: an argument for an integrated approach to violence against women. Londres : London Metropolitan University, Child and Woman Abuse Unit.

Krane, J., Oxman-Martinez, J. et Ducey, K. (2000). Violence against women and ethnoracial minority women: examining assumptions about ethnicity and "race". Canadian ethnic studies, 32(3), 1-18.

Kukathas, C. (2001). Is feminism bad for multiculturalism? Public affairs quarterly, 15(2), 83-98.

Manjarrés, N. (2007). Critical discourse analysis: a review of the critique. Lenguaje, 35(1), 219-242.

McGuigan, J. (2011). Apprentices to cool capitalism. Dans J. Richardson et J. Burridge (dir.), Analysing media discourse (p. 35-45). New York : Routeledge.

Musselin, C. (2000). The role of ideas in the emergence of convergent higher education policies in Europe: the case of France. Cambridge, Mass : Harvard University, Center for European Studies,. (Working Paper Series $\mathrm{n}^{\circ}$ 73)

Okin, S. (1997). Is multiculturalisme bad for women? [en ligne]. Boston Review, 22. http://new.bostonreview.net/BR22.5/okin.html

Oxman-Martinez, J., Krane, J., Corbin, N. et Loiselle-Léonard, M. (2002). Competing conceptions of conjugal violence - insights from an intersectional framework. Montréal : Centre d'études appliquées sur la famille, Université McGill et Immigration et métropoles.

Oxman-Martinez, J. et Krane, J. (2005). Un décalage entre théorie et pratique? Violence conjugale et femmes issues des minorités ethniques. Journal international de victimologie, 3(3), 1-14.

Piché, V. Renaud, J. et Gingras, L. (2002). L'insertion économique des nouveaux immigrants dans le marché du travail à Montréal : une approche longitudinale. Population, 37(1), 63-89.

Pickering, M. (2011). Apprentices to cool capitalism. Dans J. E. Richardson et J. D. Burridge (dir.), Analysing media discourse (p. 87-98). New York : Routeledge.

Poutignat, P. et Streiff-Fenart, J. (1995). Théories de l'ethnicité. Paris : Presses universitaires de France.

Renault, E. (2004). L'expérience de l'injustice : reconnaissance et clinique de l'injustice. Paris : La Découverte.

Renault, E. (2007). What is the use of the notion of the struggle of recognition? Revista de ciencia politica, 27(2), 195-205.

Renaud, J., Piché, V. et Godin, J.-F. (2003). L'origine nationale et l'insertion économique des immigrants au cours de leurs dix premières années au Québec. Sociologie et sociétés, 35(1), 165-184.

Richardson, J. E. (2007). Analysing Newspapers. New York : Palgrave Macmillan.

Rojas Viger, C. (2008). L'impact des violences structurelle et conjugale en contexte migratoire : perceptions d'intervenants pour le contrer. Nouvelles pratiques sociales, 20(2), 124-141.

Said, E. (1978). L'Orientalisme : l’Orient créé par l'Occident. Paris : Seuil.

Siméant, J. (1998). La cause des sans-papiers, Paris : Presses de la Fondation nationale de science politique.

Sokoloff, N. et Dupont, I. (2005). Domestic violence at the intersection of race, class, and gender: challenges and contribution to understanding violence against marginalized women in diverse communities. Violence against women, 11(38), 38-64.

Alterstice - Revue Internationale de la Recherche Interculturelle, vol. $3, n^{\circ} 2$ 
Statistique Canada (2011). La violence familiale au Canada : un profil statistique. Ottawa : Statistique Canada. $\left(n^{\circ} 85-224-X\right)$

Statistique Canada (2013). La violence familiale au Canada : un profil statistique, 2011. Ottawa : Statistique Canada. $\left(n^{\circ} 85-002-X\right)$

Thompson, J. (1995). The media and modernity. A social theory of the media. Cambridge : Polity Press.

Tolan, P. Gorman-Smith, D. et Henry, D. (2006). Family violence. Annual review of psychology, 57, 557-583.

Toolan, M. (1997). What is critical discourse analysis and why are people saying such terrible things about it? Language and literature, 6(2), 83-103.

Turner, G. (2011). "The road to the lesbian nation is not an easy one" : "us" and "them" in Diva magazine. Dans J. Richardson et J. Burridge (dir.), Analysing media discourse (p. 99-110). New York : Routeledge.

Van Dijk, T. A. (2001). critical discourse analysis. Dans D. Schiffrin, D. Tannen et H. Hamilton (dir.), Handbook of discourse analysis. Malden, Mass : Blackwell.

Van Leeuwen, T. (2008). Discourse and practice: new tools for critical discourse analysis. Oxford : Oxford University Press.

Voirol, O. (2005). Les luttes pour la visibilité. Esquisse d’une problématique. Réseaux, 1(129-130), 89-121.

Wilson, C., Gutiérrez, F. et Chao, L. (2003). Racism, sexism and the media. Thousand Oaks, CA : Sage Publications.

Wodak, R. et Meyer, M. (2009). Critical discourse analysis: history, agenda, theory and methodology. Dans R. Wodak et M. Meyer (dir.), Methods of critical discourse analysis (p. 1-34). Londres : Sage Publications.

Zorbas, V. (2002). Le programme "Côté cour ». Dans J. Oxman-Martinez et N. Lapierre Vincent (dir.), Statut précaire d'immigration, dépendance et vulnérabilité des femmes à la violence : les impacts sur leur santé (p. 73-75). Montréal : Centre d'études appliquées sur la famille, Université McGill et Immigration et métropoles. 